\section{GSK renews malaria efforts}

In an effort to move new treatments for malaria into the marketplace, GlaxoSmithKline Biologicals (GSK) has formed a private-public partnership with the World Health Organisation (WHO) and a US non-profit group, Program for Appropriate Technology in Health (PATH) to fund the production of an oral treatment and the development of a vaccine for childhood malaria in sub-saharan Africa

The projects were announced last month at the same that the UK government promised tax cut incentives for pharmaceutical companies to boost and enable third world drug R\&D (see next page). However, GSK scientific officer John Horton, who's team has been developing GSK's oral malaria treatment, called LAPDAP, since 1991, says "The govern-

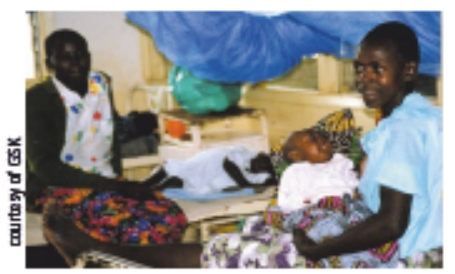

marketplace limitations of distributing drugs to third world countries: "For world access to these drugs we must operate in collaboration with public organizations such as WHO, who have the expertise to distribute in third world countries." Winston Gutteridge of the WHO adds, "GlaxoWellcome had a long history in malaria research and so too did SmithKline Beecham.

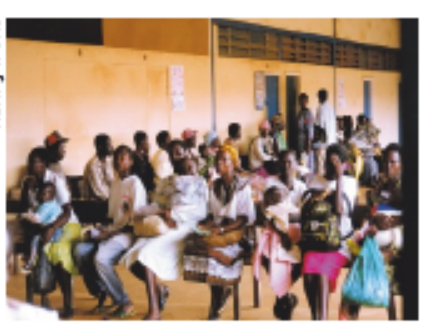
Outpatients clinic in a Kenyan hospital acute uncomplicated malaria in children. LAPDAP may prove a suitable replacement for the drug sulfadoxine/pyrimethamine (SP), as parasite resistance to this therapy is increasing worldwide. LAPDAP is intended for release in March 2002.

The PATH-backed malaria vacine is in Phase II clinical trials and has shown a $70 \%$ success rate against Plasmodium falctparum in a group of healthy young Gambian men. Earliest release could be 2007, following 17 These two companies had the most exciting research and development portfolios in this area and when they merged they were the most obvious company to back."

Discovered by scientists at Liverpool University in 1986, LAPDAP is a combination of two existing ment's tax promises to stimulate drug development in the developing world are behind GSK's and WHO's plans by about 15 years."

According to GSK spokesperson, Pamela Duncan, public-private partnerships will allow the company to overcome former

malaria drugs, chlorproguanil (marketed by ICI as Lapurdine in the 1970s) and Dapsone (also used for leprosy treatment), hence its cost of production is low at less than one US dollar a tablet. The two drugs work synergistically on the folate pathway in the parasite and are intended to treat

\section{Osteoporosis drugs show promise against malaria}

Drugs that have already been approved for treatment of osteoporosis may form the basis of the next generation of ant malarial compounds, according to a team of British, American and Venezuelan researchers. In addition to their activity against Plasmodium falciparum, a causative agent of malaria, the compounds, called bisphosphonates, appear to kill trypanosomes, Leishmanta, and Taxoplasma gondi1. Together, these parasites infect over 500 million people annually worldwide, mostly in the tropics.

The researchers, whose work appears in the 15 March issue of the Journal of Medicinal Chemistry, had previously performed structural studies on protozoan parasites and discovered large amounts of pyrophosphate stored in organelles which they named acidocalcisomes. "We found it logical to test hydrolytically stable analogs of pyrophosphate - bisphosphonates - against the parasites, and were encouraged by the anti-parasitic activity we found," says study author Roberto Docampo from the University of
Illinois Urbana-Champagne. Docampo adds, "organelles similar to acidocalcisomes, called volutin granules, have been described in bacteria and fungi,"

trypanosome showing acidocalcisome organelles

suggesting that the bisphosphonates may be useful against a wide range of pathogens.

Bisphosphonates already approved for human use include Merck's Fosamax, Procter and Gamble's Actonel, and Novartis' Aredia. Fosamax, which has been prescribed to over 3 million patients since its introduction in 1995 , is currently the leading osteoporosis drug and enjoys annual worldwide sales of over \$1 billion.

Having demonstrated the potential of bisphosphonates against parasites in vitro, the researchers are now testing the years of development by GSK. The WHO comments, "There are over 100 malaria vaccines being developed but GSK's is the most advanced at the moment."

PATH spokesperson, Gina Rabinovich says, "Our 6.7 million US dollar partnership with GSK is not a blank check. We are in partnership with other vaccine developments in the US, Australia and India but we still do not know which candidate moves the vacine forward first. These vaccines are very expensive to make and drug companies know they are not going to make big profits with them as they could in the European market."

Georgina Kenyon, London

drugs in animal models of parasitic diseases. Docampo says that the animal results have been promising so far, particularly against visceral and cutaneous leishmaniasis.

Eric Oldfield, a University of Illinois researcher and co-author, states that "new drugs are urgently required in the less developed nations, since the parasites are becoming drug resistant at an alarming rate." So far, however, the researchers have not determined how readily parasites will develop resistance to bisphosphonates.

Another concern is that, while Fosamax and its relatives have been blockbuster drugs for treating osteoporosis in developed countries, the drugs' prices remain out of reach for the developing world, where parasitic diseases are concentrated. Still, Docampo is hopeful that pharmaceutical companies will be willing to offer discounts to poor countries: "The synthesis of these drugs is not expensive. This could be a case where the intervention of charitable organizations could have a major role." Alan Dove, Philadelphia 